\title{
CONVERGENCE AND ALMOST SURE T-STABILITY FOR RANDOM NOOR-TYPE ITERATIVE SCHEME
}

\author{
Godwin Amechi Okeke ${ }^{1 \S}$, Kanayo Stella $\mathrm{Eke}^{2}$ \\ ${ }^{1}$ Department of Mathematics \\ Michael Okpara University of Agriculture \\ Umudike, P.M.B. 7267, Umuahia, Abia State, NIGERIA \\ ${ }^{2}$ Department of Mathematics \\ College of Science and Technology \\ Covenant University \\ Canaanland, KM 10 Idiroko Road, P.M.B. 1023 Ota \\ Ogun State, NIGERIA
}

\begin{abstract}
The purpose of this study is to introduce a Noor-type random iterative scheme and prove the convergence of this kind of random iterative scheme for certain $\phi$-weakly contractive type random operators. The Bochner integrability of random fixed points for this kind of random operators and the almost sure $T$-stability and convergence for Noor-type random iterative scheme are established. Our results are stochastic generalizations of the deterministic fixed point theorems of Berinde [7, 8] and Rhoades [29]-[32].
\end{abstract}

AMS Subject Classification: 47H09, 47H10, 49M05, 54H25

Key Words: Bochner integrability, Noor-type random iterative scheme, almost sure $T$ stability, random fixed point

\section{Introduction}

Probabilistic functional analysis has emerged over the years as one of the mo-

Received: June 12, 2015

Published: March 21, 2016

${ }^{\S}$ Correspondence author (c) 2016 Academic Publications, Ltd.

url: www.acadpubl.eu 
mentous mathematical disciplines in view of its requirements in dealing with probabilistic models in applied problems. Random fixed point theorems are stochastic generalizations of classical or deterministic fixed point theorems and are required for the theory of random equations, random matrices, random partial differential equations and various kinds of random operators arising in physical systems (see, e.g. Joshi and Bose [18], Zhang [36]). The theory of random fixed point theorems was initiated in 1950s by Prague school of probabilists. After the classical results of Bharucha-Reid [9] in 1976, where He gave sufficient conditions for a stochastic analogue of Schauder's fixed point theorem for random operators, the theory of random fixed points received unprecedented attention by several researchers and many ineresting results have appeared in literature (see, e.g., [4], [6], [10], [17], [18], [20], [22], [26], [27], [33], [35], [37]). Spacek [34] and Hans [14] established stochastic analogue of the Banach fixed point theorem in a separable metric space. Itoh [17] in 1979 generalized and extended Spacek and Han's theorem to a multi-valued contraction random operator. Papageorgiou [27] proved several random fixed point theorems for measurable closed and nonclosed valued multifunctions satisfying general continuity conditions. His results improves earlier results announced by Engl [12], Itoh [17] and Reich [28]. Xu [35] in 1990 extended the results of Itoh to a nonself-random operator $T$, where $T$ satisfies either weakly inward or the Leray-Schauder conditon (see Xu [35]). Shahzad and Latif [33] in 1999 proved a general random fixed point theorem for continuous random operators. As applications, they derived a number of random fixed points theorems for various classes of 1-set and 1-ball contractive random operators (e.g., operators of contractive type with compact or completely continuous perturbations, operators of semicontractive type, etc.). Arunchai and Plubtieng [4] established some random fixed point theorem for the sum of a weakly-strongly continuous random operator and a nonexpansive random operator in Banach spaces. Their results are the random versions of some deterministic fixed point theorems of Edmunds [11] and O'Regan [26]. Chang et al. [10] and Beg and Abbas [6] proved some convergence theorems of random Ishikawa schemes and random Mann iterative schemes for strongly pseudo-contractive operators and contraction operatos respectively, in separable reflexive Banach spaces. Recently, Zhang et al. [37] studied the almost sure $T$-stability and convergence of Ishikawa-type and Mann-type random algotithms for certain $\phi$-weakly contractive type random operators in a separable Banach space. They established the Bochner integrability of random fixed point for this kind of random operators and the almost sure $T$-stability and convergence for these two kinds of random iterative algorithms under suitable conditions. Their classical results extends 
several known related results in literature (see, e.g. Berinde [7], Olatinwo [25], Rhoades [30, 31], Olaleru and Mogbademu [24], Akewe and Okeke [1], Akewe et al. [2], Akewe and Okeke [3] among others).

In 1953, Mann [19] introduced the Mann iterative scheme and used it to prove the convergence of the sequence to the fixed points for which the Banach principle is not applicable. Later in 1974, Ishikawa [16] introduced an iterative process to obtain the convergence of a Lipschitzian pseudocontractive operator when Mann iterative scheme failed to converge. In 2000 Noor [21] gave the following three-step iterative scheme (or Noor iteration) for solving nonlinear operator equations in uniformly smooth Banach spaces.

Let $D$ be a nonempty convex subset of a Banach space $E$ and let $T: D \rightarrow D$ be a mapping. For a given $x_{0} \in D$, compute the sequence $\left\{x_{n}\right\}_{n=0}^{\infty}$ by the iterative schemes

$$
\left\{\begin{aligned}
x_{n+1} & =\left(1-\alpha_{n}\right) x_{n}+\alpha_{n} T y_{n}, \\
y_{n} & =\left(1-\beta_{n}\right) x_{n}+\beta_{n} T z_{n}, \\
z_{n} & =\left(1-\gamma_{n}\right) x_{n}+\gamma_{n} T x_{n}, \quad n \geq 0
\end{aligned}\right.
$$

where $\left\{\alpha_{n}\right\}_{n=0}^{\infty},\left\{\beta_{n}\right\}_{n=0}^{\infty}$ and $\left\{\gamma_{n}\right\}_{n=0}^{\infty}$ are three real sequences in $[0,1]$ satisfying some conditions.

If $\gamma_{n}=0$ and $\beta_{n}=0$, for each $n \in \mathbb{Z}, n \geq 0$, then (1.1) reduces to the iterative scheme

$$
x_{n+1}=\left(1-\alpha_{n}\right) x_{n}+\alpha_{n} T x_{n}, \quad n \in \mathbb{Z}, n \geq 0,
$$

which is called the one-step (or Mann iterative scheme), introduced by Mann [19].

For $\gamma_{n}=0,(1.1)$ reduces to:

$$
\left\{\begin{aligned}
x_{n+1} & =\left(1-\alpha_{n}\right) x_{n}+\alpha_{n} T y_{n}, \\
y_{n} & =\left(1-\beta_{n}\right) x_{n}+\beta_{n} T x_{n}, \quad n \geq 0
\end{aligned}\right.
$$

where $\left\{\alpha_{n}\right\}_{n=0}^{\infty}$ and $\left\{\beta_{n}\right\}_{n=0}^{\infty}$ are two real sequences in [0,1] satisfying some conditions. Equation (1.3) is called the two-step (or Ishikawa iterative process) introduced by Ishikawa [16].

In 1989, Glowinski and Le-Tallec [13] used a three-step iterative process to solve elastoviscoplasticity, liquid crystal and eigenvalue problems. They established that three-step iterative scheme performs better than one-step (Mann) and two-step (Ishikawa) iterative schemes. Haubruge et al. [15] studied the convergence analysis of the three-step iterative processes of Glowinski and LeTallec [13] and used the three-step iteration to obtain some new splitting type 
algorithms for solving variational inequalities, separable convex programming and minimization of a sum of convex functions. They also proved that three-step iteration also lead to highly parallelized algorithms under certain conditions. Hence, we can conclude by observing that three-step iterative scheme play an important role in solving various problems in pure and applied sciences.

The purpose of this study is to introduce Noor-type random iterative scheme and study the almost sure $T$-stability and convergence of our newly introduced Noor-type random iterative scheme for certain $\phi$-weakly contractive type random operators in a separable Banach space. We shall prove the Bochner integrability of random fixed points for this kind of random operators and establish the almost sure $T$-stability and convergence for our newly introduced Noor-type random algorithms. Our results are stochastic generalizations of the deterministic fixed point theorems of Berinde [7, 8], Rhoades [29]-[32] among others.

\section{Preliminaries}

Throughout this study, we assume that $(\Omega, \xi, \mu)$ is a complete probability measure space and $E$ is a nonempty subset of a separable Banach space $X$.

The following definitions will be needed in this study.

Definition 2.1. (see [18]) A random variable $x(\omega)$ is Bochner integrable if $\|x(\omega)\| \in L^{1}(\Omega ; \xi ; \mu)$, i.e.,

$$
\int_{\Omega}\|x(\omega)\| d \mu(\omega)<\infty
$$

Proposition 2.2. (see [18]) A random variable $x(\omega)$ is Bochner integrable if and only if there exists a sequence of random variables $\left\{x_{n}(\omega)\right\}_{n=1}^{\infty}$ converging strongly to $x(\omega)$ almost surely (a.s.) such that

$$
\lim _{n \rightarrow \infty} \int_{\Omega}\left\|x_{n}(\omega)-x(\omega)\right\| d \mu(\omega)=0 .
$$

Definition 2.3. (see [37]) Let $(\Omega, \zeta, \mu)$ be a complete probability measure space and $E$ be a nonempty subset of a separable Banach space $X$. Let $T: \Omega \times$ $E \rightarrow E$ be a random operator. Denote by $F(T)=\left\{x^{*}(\omega) \in E: T\left(\omega, x^{*}(\omega)\right)=\right.$ $\left.x^{*}(\omega), \omega \in \Omega\right\}$ the random fixed point set of $T$. For any given random variable $x_{0}(\omega) \in E$, define an iterative scheme $\left\{x_{n}(\omega)\right\}_{n=0}^{\infty} \subset E$ by

$$
x_{n+1}(\omega)=f\left(T ; x_{n}(\omega)\right), \quad n=0,1,2, \cdots,
$$


where $f$ is some function measurable in the second variable.

Let $x^{*}(\omega)$ be a random fixed point of $T$ and Bochner integrable with respect to $\left\{x_{n}\right\}_{n=0}^{\infty}$. Let $\left\{y_{n}(\omega)\right\}_{n=0}^{\infty} \subset E$ be an arbitrary sequence of a random variable. Denote

$$
\varepsilon_{n}(\omega)=\left\|y_{n+1}(\omega)-f\left(T ; y_{n}(\omega)\right)\right\|,
$$

and assume that $\left\|\varepsilon_{n}(\omega)\right\| \in L^{1}(\Omega(\xi, \mu) \quad(n=0,1, \cdots)$. Then, the iterative scheme (2.3) is $T$-stable almost surely (a.s.) (or the iterative scheme (2.3) is stable with respect to $T$ almost surely) if and only if

$$
\lim _{n \rightarrow \infty} \int_{\Omega}\left\|\varepsilon_{n}(\omega)\right\| d \mu(\omega)=0
$$

implies that $x^{*}(\omega)$ is Bochner integrable with respect to $\left\{y_{n}(\omega)\right\}_{n=0}^{\infty}$.

Definition 2.4. (see [37]) Let $(\Omega, \xi, \mu)$ be a complete probability measure space and $E$ be a nonempty subset of a separable Banach space $X$. A random operator $T: \Omega \times E \rightarrow E$ is the $\phi$-weakly contractive type if there exists a continuous and nondecreasing function $\phi: \mathbb{R}^{+} \rightarrow \mathbb{R}^{+}$with $\phi(t)>0(\forall t \in$ $(0, \infty))$ and $\phi(0)=0$ such that $\forall x, y \in E, \omega \in \Omega$,

$$
\int_{\Omega}\|T(\omega, x)-T(\omega, y)\| d \mu(\omega) \leq \int_{\Omega}\|x-y\| d \mu(\omega)-\phi\left(\int_{\Omega}\|x-y\| d \mu(\omega)\right) .
$$

The purpose of this study is to introduce the Noor-type random iterative scheme for certain random operators $T: \Omega \times E \rightarrow E$ of the $\phi$-weakly contractive type in separable Banach space $(E,\|\cdot\|)$.

Let $(\Omega, \xi, \mu)$ be a complete probability measure space and $E$ is a nonempty subset of a separable Banach space $X$ and let $T: \Omega \times E \rightarrow E$ be a random operator. For a given $x_{0}(\omega) \in E$, compute the sequence $\left\{x_{n}(\omega)\right\}_{n=0}^{\infty}$ by the iterative schemes

$$
\left\{\begin{aligned}
x_{n+1}(\omega) & =\left(1-\alpha_{n}\right) x_{n}(\omega)+\alpha_{n} T\left(\omega, y_{n}(\omega)\right) \\
y_{n}(\omega) & =\left(1-\beta_{n}\right) x_{n}(\omega)+\beta_{n} T\left(\omega, z_{n}(\omega)\right), \\
z_{n}(\omega) & =\left(1-\gamma_{n}\right) x_{n}(\omega)+\gamma_{n} T\left(\omega, x_{n}(\omega)\right), \quad n \geq 0
\end{aligned}\right.
$$

where $\left\{\alpha_{n}\right\},\left\{\beta_{n}\right\}$ and $\left\{\gamma_{n}\right\}$ are real sequences in $(0,1)$.

Observe that if $\gamma_{n} \equiv 0$ for each $n \in \mathbb{N}$ in $(2.7)$, then we have the Ishikawatype random iterative scheme, introduced by Zhang et al. [37].

$$
\left\{\begin{aligned}
x_{n+1}(\omega) & =\left(1-\alpha_{n}\right) x_{n}(\omega)+\alpha_{n} T\left(\omega, y_{n}(\omega)\right), \\
y_{n}(\omega) & =\left(1-\beta_{n}\right) x_{n}(\omega)+\beta_{n} T\left(\omega, x_{n}(\omega)\right), \quad n \geq 0
\end{aligned}\right.
$$


where $\left\{\alpha_{n}\right\}$ and $\left\{\beta_{n}\right\}$ are real sequences in $(0,1)$.

If $\beta_{n}=\gamma_{n} \equiv 0$ for each $n \in \mathbb{N}$ in (2.7), then we obtain the Mann-type random iterative scheme, introduced by Zhang et al. [33].

$$
\left\{x_{n+1}(\omega)=\left(1-\alpha_{n}\right) x_{n}(\omega)+\alpha_{n} T\left(\omega, x_{n}(\omega)\right), \quad n \geq 0\right.
$$

where $\left\{\alpha_{n}\right\}$ is real sequences in $(0,1)$.

We shall establish that under suitable conditions that the random fixed point of $\phi$-weakly contractive type random operators is Bochner integrable. Our results are the random versions of some deterministic fixed point theorems of Berinde [7], [8] and Rhoades [29]-[32]. Hence, it generalizes and improves the results of these authors and several other known results in literature (see, e.g, Akewe and Okeke [1], Olatinwo [25], Rhoades [30, 31], Olaleru and Mogbademu [24], Zhang et al. [37]).

The following lemma will be needed in this study.

Lemma 2.5. (see [5]) Let $\left\{\zeta_{n}\right\}$ and $\left\{\lambda_{n}\right\}$ be two sequences of nonnegative real numbers. Let $\left\{\sigma_{n}\right\}$ be a sequence of positive numbers satisfying the conditions: $\sum_{n=1}^{\infty} \sigma_{n}=\infty$ and $\lim _{n \rightarrow \infty} \frac{\zeta_{n}}{\sigma_{n}}=0$. If the following condition is satisfied:

$$
\lambda_{n+1} \leq \lambda_{n}-\sigma_{n} \phi\left(\lambda_{n}\right)+\zeta_{n}, \quad \forall n \geq 1
$$

where $\phi: \mathbb{R}^{+} \rightarrow \mathbb{R}^{+}$is a continuous and strictly increasing function with $\phi(0)=0$, then $\left\{\lambda_{n}\right\}$ converges to 0 as $n \rightarrow \infty$.

\section{Main Results}

Theorem 3.1. Let $(E,\|\|$.$) be a separable Banach space, T: \Omega \times E \rightarrow E$ be a random operator of the $\phi$-weakly contractive type with $F(T) \neq \emptyset, x^{*}(\omega)$ be a random fixed point of $T$, and $\left\{x_{n}(\omega)\right\}$ be a Noor-type random iterative sequence as defined in (2.7) where $\left\{\alpha_{n}\right\},\left\{\beta_{n}\right\}$ and $\left\{\gamma_{n}\right\}$ are real sequences in $(0,1)$ such that $\sum_{n=1}^{\infty} \alpha_{n} \beta_{n} \gamma_{n}=\infty$. Then, the random fixed point $x^{*}(\omega)$ of $T$ is Bochner integrable.

Proof. It suffices to show that

$$
\lim _{n \rightarrow \infty} \int_{\Omega}\left\|x_{n}(\omega)-x^{*}(\omega)\right\| d \mu(\omega)=0
$$


Using (2.6) and (2.7), we have:

$$
\begin{aligned}
\int_{\Omega}\left\|x_{n+1}(\omega)-x^{*}(\omega)\right\| d \mu(\omega) \leq & \left(1-\alpha_{n}\right) \int_{\Omega}\left\|x_{n}(\omega)-x^{*}(\omega)\right\| d \mu(\omega) \\
& +\alpha_{n} \int_{\Omega}\left\|T\left(\omega, y_{n}(\omega)\right)-x^{*}(\omega)\right\| d \mu(\omega) \\
\leq & \left(1-\alpha_{n}\right) \int_{\Omega}\left\|x_{n}(\omega)-x^{*}(\omega)\right\| d \mu(\omega) \\
& +\alpha_{n}\left[\int_{\Omega}\left\|y_{n}(\omega)-x^{*}(\omega)\right\| d \mu(\omega)\right. \\
& \left.-\phi\left(\int_{\Omega}\left\|y_{n}(\omega)-x^{*}(\omega)\right\| d \mu(\omega)\right)\right] \\
\leq & \left(1-\alpha_{n}\right) \int_{\Omega}\left\|x_{n}(\omega)-x^{*}(\omega)\right\| d \mu(\omega) \\
& +\alpha_{n} \int_{\Omega}\left\|y_{n}(\omega)-x^{*}(\omega)\right\| d \mu(\omega) .
\end{aligned}
$$

Similarly,

$$
\begin{aligned}
\int_{\Omega}\left\|y_{n}(\omega)-x^{*}(\omega)\right\| d \mu(\omega) \leq & \left(1-\beta_{n}\right) \int_{\Omega}\left\|x_{n}(\omega)-x^{*}(\omega)\right\| d \mu(\omega) \\
& +\beta_{n} \int_{\Omega}\left\|T\left(\omega, z_{n}(\omega)\right)-x^{*}(\omega)\right\| d \mu(\omega) \\
\leq & \left(1-\beta_{n}\right) \int_{\Omega}\left\|x_{n}(\omega)-x^{*}(\omega)\right\| d \mu(\omega) \\
& +\beta_{n}\left[\int_{\Omega}\left\|z_{n}(\omega)-x^{*}(\omega)\right\| d \mu(\omega)\right. \\
& \left.-\phi\left(\int_{\Omega}\left\|z_{n}(\omega)-x^{*}(\omega)\right\| d \mu(\omega)\right)\right] \\
\leq & \left(1-\beta_{n}\right) \int_{\Omega}\left\|x_{n}(\omega)-x^{*}(\omega)\right\| d \mu(\omega) \\
& +\beta_{n} \int_{\Omega}\left\|z_{n}(\omega)-x^{*}(\omega)\right\| d \mu(\omega) .
\end{aligned}
$$

Also, 


$$
\begin{aligned}
\int_{\Omega}\left\|z_{n}(\omega)-x^{*}(\omega)\right\| d \mu(\omega) \leq & \left(1-\gamma_{n}\right) \int_{\Omega}\left\|x_{n}(\omega)-x^{*}(\omega)\right\| d \mu(\omega) \\
& +\gamma_{n} \int_{\Omega}\left\|T\left(\omega, x_{n}(\omega)\right)-x^{*}(\omega)\right\| d \mu(\omega) \\
\leq & \left(1-\gamma_{n}\right) \int_{\Omega}\left\|x_{n}(\omega)-x^{*}(\omega)\right\| d \mu(\omega) \\
& +\gamma_{n}\left[\int_{\Omega}\left\|x_{n}(\omega)-x^{*}(\omega)\right\| d \mu(\omega)\right. \\
& \left.-\phi\left(\int_{\Omega}\left\|x_{n}(\omega)-x^{*}(\omega)\right\| d \mu(\omega)\right)\right] \\
= & \int_{\Omega}\left\|x_{n}(\omega)-x^{*}(\omega)\right\| d \mu(\omega) \\
& -\gamma_{n} \phi\left(\int_{\Omega}\left\|x_{n}(\omega)-x^{*}(\omega)\right\| d \mu(\omega)\right) .
\end{aligned}
$$

Using (3.4) in (3.3), we obtain:

$$
\begin{aligned}
\int_{\Omega}\left\|y_{n}(\omega)-x^{*}(\omega)\right\| d \mu(\omega) \leq & \left(1-\beta_{n}\right) \int_{\Omega}\left\|x_{n}(\omega)-x^{*}(\omega)\right\| d \mu(\omega) \\
& +\beta_{n}\left[\int_{\Omega}\left\|x_{n}(\omega)-x^{*}(\omega)\right\| d \mu(\omega)\right. \\
& \left.-\gamma_{n} \phi\left(\int_{\Omega}\left\|x_{n}(\omega)-x^{*}(\omega)\right\| d \mu(\omega)\right)\right] \\
= & \int_{\Omega}\left\|x_{n}(\omega)-x^{*}(\omega)\right\| d \mu(\omega) \\
& -\beta_{n} \gamma_{n} \phi\left(\int_{\Omega}\left\|x_{n}(\omega)-x^{*}(\omega)\right\| d \mu(\omega)\right) .
\end{aligned}
$$

Using (3.5) in (3.2), we have

$$
\begin{aligned}
\int_{\Omega}\left\|x_{n+1}(\omega)-x^{*}(\omega)\right\| d \mu(\omega) \leq & \left(-\alpha_{n}\right) \int_{\Omega}\left\|x_{n}(\omega)-x^{*}(\omega)\right\| d \mu(\omega) \\
& +\alpha_{n}\left[\int_{\Omega}\left\|x_{n}(\omega)-x^{*}(\omega)\right\| d \mu(\omega)\right. \\
& \left.-\beta_{n} \gamma_{n} \phi\left(\int_{\Omega}\left\|x_{n}(\omega)-x^{*}(\omega)\right\| d \mu(\omega)\right)\right] \\
= & \int_{\Omega}\left\|x_{n}(\omega)-x^{*}(\omega)\right\| d \mu(\omega) \\
& -\alpha_{n} \beta_{n} \gamma_{n} \phi\left(\int_{\Omega}\left\|x_{n}(\omega)-x^{*}(\omega)\right\| d \mu(\omega)\right) .
\end{aligned}
$$


If we take $\lambda_{n}=\int_{\Omega}\left\|x_{n}(\omega)-x^{*}(\omega)\right\| d \mu(\omega), \sigma_{n}=\alpha_{n} \beta_{n} \gamma_{n}$ and $\zeta_{n}=0$ in Lemma 2.5. By the conditions of Theorem 3.1, we see that the conditions of Lemma 2.5 are satisfied. Hence, we have

$$
\lim _{n \rightarrow \infty} \int_{\Omega}\left\|x_{n}(\omega)-x^{*}(\omega)\right\| d \mu(\omega)=0
$$

The proof of Theorem 3.1 is completed.

We now obtain the following corollary which are the results of Zhang et al. [37] as a consequence of Theorem 3.1.

Corollary 3.2. Let $(E,\|\|$.$) be a separable Banach space, T: \Omega \times E \rightarrow E$ be a random operator of the $\phi$-weakly contractive type with $F(T) \neq \emptyset, x^{*}(\omega)$ be a random fixed point of $T$, and $\left\{x_{n}(\omega)\right\}$ be an Ishikawa-type random iterative sequence as defined in (2.8) where $\left\{\alpha_{n}\right\}$ and $\left\{\beta_{n}\right\}$ are real sequences in $(0,1)$ such that $\sum_{n=1}^{\infty} \alpha_{n} \beta_{n}=\infty$. Then, the random fixed point $x^{*}(\omega)$ of $T$ is Bochner integrable.

Corollary 3.3. Let $(E,\|\|$.$) be a separable Banach space, T: \Omega \times E \rightarrow E$ be a random operator of the $\phi$-weakly contractive type with $F(T) \neq \emptyset, x^{*}(\omega)$ be a random fixed point of $T$, and $\left\{x_{n}(\omega)\right\}$ be a Mann-type random iterative sequence as defined in (2.9) where $\left\{\alpha_{n}\right\}$ is a real sequence in $(0,1)$ such that $\sum_{n=1}^{\infty} \alpha_{n}=\infty$. Then, the random fixed point $x^{*}(\omega)$ of $T$ is Bochner integrable.

Theorem 3.4. Let $(E,\|\|$.$) be a separable Banach space and T: \Omega \times E \rightarrow E$ be a random operator of the $\phi$-weakly contractive type with $F(T) \neq \emptyset$. Let $x^{*}(\omega)$ be a random fixed point of $T$. Let $\left\{x_{n}(\omega)\right\}_{n=0}^{\infty}$ be a Noor-type random iterative sequence as defined in (2.7) converging strongly to $x^{*}(\omega)$ almost surely, where $\left\{\alpha_{n}\right\},\left\{\beta_{n}\right\}$ and $\left\{\gamma_{n}\right\}$ are real sequences in $(0,1)$ such that $0<\alpha \leq \alpha_{n}$, $0<\beta \leq \beta_{n}$ and $0<\gamma \leq \gamma_{n}$. Then, $\left\{x_{n}\right\}_{n=0}^{\infty}$ is T-stable almost surely. and

Proof. Let $\left\{m_{n}(\omega)\right\}_{n=0}^{\infty}$ be an arbitrary sequence of random variables in $E$

$$
\left\|\varepsilon_{n}(\omega)\right\|=\left\|m_{n+1}-\left(1-\alpha_{n}\right) m_{n}(\omega)-\alpha_{n} T\left(\omega, k_{n}(\omega)\right)\right\|, \quad n=0,1, \cdots
$$

where $k_{n}(\omega)=\left(1-\beta_{n}\right) m_{n}(\omega)+\beta_{n} T\left(\omega, z_{n}(\omega)\right)$ and $\lim _{n \rightarrow \infty} \int_{\Omega}\|\varepsilon(\omega)\| d \mu(\omega)=0$. Next, we prove that $x^{*}(\omega)$ is Bochner integrable with respect to the sequence 
$\left\{m_{n}(\omega)\right\}_{n=0}^{\infty}$. Using (3.8), we have

$$
\begin{aligned}
\int_{\Omega}\left\|m_{n+1}(\omega)-x^{*}(\omega)\right\| d \mu(\omega) \leq & \int_{\Omega} \| m_{n+1}(\omega)-\left(1-\alpha_{n}\right) m_{n}(\omega) \\
& -\alpha_{n} T\left(\omega, k_{n}(\omega)\right) \| d \mu(\omega) \\
& +\left(1-\alpha_{n}\right) \int_{\Omega}\left\|m_{n}(\omega)-x^{*}(\omega)\right\| d \mu(\omega) \\
& +\alpha_{n} \int_{\Omega}\left\|T\left(\omega, k_{n}(\omega)\right)-x^{*}(\omega)\right\| d \mu(\omega) \\
= & \int_{\Omega}\left\|\varepsilon_{n}(\omega)\right\| d \mu(\omega) \\
& +\left(1-\alpha_{n}\right) \int_{\Omega}\left\|m_{n}(\omega)-x^{*}(\omega)\right\| d \mu(\omega) \\
& +\alpha_{n} \int_{\Omega}\left\|T\left(\omega, k_{n}(\omega)\right)-x^{*}(\omega)\right\| d \mu(\omega) .
\end{aligned}
$$

Using (2.6), we have

$$
\begin{aligned}
& \int_{\Omega}\left\|T\left(\omega, k_{n}(\omega)\right)-x^{*}(\omega)\right\| d \mu(\omega) \leq \int_{\Omega}\left\|k_{n}(\omega)-x^{*}(\omega)\right\| d \mu(\omega) \\
& -\phi\left(\int_{\Omega}\left\|k_{n}(\omega)-x^{*}(\omega)\right\| d \mu(\omega)\right) \\
\leq & \int_{\Omega}\left\|k_{n}(\omega)-x^{*}(\omega)\right\| d \mu(\omega) \\
\leq & \left(1-\beta_{n}\right) \int_{\Omega}\left\|m_{n}(\omega)-x^{*}(\omega)\right\| d \mu(\omega)+\beta_{n}\left[\int_{\Omega}\left\|T\left(\omega, z_{n}(\omega)\right)-x^{*}(\omega)\right\| d \mu(\omega)\right] \\
\leq & \left(1-\beta_{n}\right) \int_{\Omega}\left\|m_{n}(\omega)-x^{*}(\omega)\right\| d \mu(\omega)+\beta_{n}\left[\int_{\Omega}\left\|z_{n}(\omega)-x^{*}(\omega)\right\| d \mu(\omega)\right. \\
& \left.-\phi\left(\int_{\Omega}\left\|z_{n}(\omega)-x^{*}(\omega)\right\| d \mu(\omega)\right)\right] \\
\leq & \left(1-\beta_{n}\right) \int_{\Omega}\left\|m_{n}(\omega)-x^{*}(\omega)\right\| d \mu(\omega)+\beta_{n} \int_{\Omega}\left\|z_{n}(\omega)-x^{*}(\omega)\right\| d \mu(\omega)
\end{aligned}
$$




$$
\begin{aligned}
\leq & \left(1-\beta_{n}\right) \int_{\Omega}\left\|m_{n}(\omega)-x^{*}(\omega)\right\| d \mu(\omega) \\
& +\beta_{n}\left[\left(1-\gamma_{n}\right) \int_{\Omega}\left\|m_{n}(\omega)-x^{*}(\omega)\right\| d \mu(\omega)+\gamma_{n} \int_{\Omega}\left\|T\left(\omega, m_{n}(\omega)\right)-x^{*}(\omega)\right\| d \mu(\omega)\right] \\
\leq & \left(1-\beta_{n}\right) \int_{\Omega}\left\|m_{n}(\omega)-x^{*}(\omega)\right\| d \mu(\omega)+\beta_{n}\left[\left(1-\gamma_{n}\right) \int_{\Omega}\left\|m_{n}(\omega)-x^{*}(\omega)\right\| d \mu(\omega)\right. \\
& \left.+\gamma_{n}\left\{\int_{\Omega}\left\|m_{n}(\omega)-x^{*}(\omega)\right\| d \mu(\omega)-\phi\left(\int_{\Omega}\left\|m_{n}(\omega)-x^{*}(\omega)\right\| d \mu(\omega)\right)\right\}\right] \\
= & \int_{\Omega}\left\|m_{n}(\omega)-x^{*}(\omega)\right\| d \mu(\omega)-\beta_{n} \gamma_{n} \phi\left(\int_{\Omega}\left\|m_{n}(\omega)-x^{*}(\omega)\right\| d \mu(\omega)\right) .
\end{aligned}
$$

Using (3.10) in (3.9), we have

$$
\begin{aligned}
\int_{\Omega}\left\|m_{n+1}(\omega)-x^{*}(\omega)\right\| d \mu(\omega) \leq & \int_{\Omega}\left\|\varepsilon_{n}(\omega)\right\| d \mu(\omega) \\
& +\left(1-\alpha_{n}\right) \int_{\Omega}\left\|m_{n}(\omega)-x^{*}(\omega)\right\| d \mu(\omega) \\
& +\alpha_{n}\left[\int_{\Omega}\left\|m_{n}(\omega)-x^{*}(\omega)\right\| d \mu(\omega)\right. \\
& \left.-\beta_{n} \gamma_{n} \phi\left(\int_{\Omega}\left\|m_{n}(\omega)-x^{*}(\omega)\right\| d \mu(\omega)\right)\right] \\
= & \int_{\Omega}\left\|\varepsilon_{n}(\omega)\right\| d \mu(\omega) \\
& +\int_{\Omega}\left\|m_{n}(\omega)-x^{*}(\omega)\right\| d \mu(\omega) \\
& -\alpha_{n} \beta_{n} \gamma_{n} \phi\left(\int_{\Omega}\left\|m_{n}(\omega)-x^{*}(\omega)\right\| d \mu(\omega)\right) .
\end{aligned}
$$

Using the assumptions that $\lim _{n \rightarrow \infty} \int_{\Omega}\|\varepsilon(\omega)\| d \mu(\omega)=0$ and $0<\alpha \leq \alpha_{n}$, $0<\beta \leq \beta_{n}, 0<\gamma \leq \gamma_{n}(\forall n \geq 1)$, we obtain

$$
\lim _{n \rightarrow \infty} \frac{\int_{\Omega}\left\|\varepsilon_{n}(\omega)\right\| d \mu(\omega)}{\alpha_{n} \beta_{n} \gamma_{n}} \leq \frac{\int_{\Omega}\left\|\varepsilon_{n}(\omega)\right\| d \mu(\omega)}{\alpha \beta \gamma}=0 .
$$

By Lemma 2.5, we take $\lambda_{n}=\int_{\Omega}\left\|m_{n}(\omega)-x^{*}(\omega)\right\| d \mu(\omega), \sigma_{n}=\alpha_{n} \beta_{n} \gamma_{n}$ and $\zeta_{n}=\int_{\Omega}\left\|\varepsilon_{n}(\omega)\right\| d \mu(\omega)$. Clearly, all the conditions of Lemma 2.5 are satisfied. Hence, we obtain

$$
\lim _{n \rightarrow \infty} \int_{\Omega}\left\|m_{n}(\omega)-x^{*}(\omega)\right\| d \mu(\omega)=0 .
$$


Conversely, if $x^{*}(\omega)$ is Bochner integrable with respect to the sequence $\left\{m_{n}(\omega)\right\}_{n=1}^{\infty}$, we obtain:

$$
\begin{aligned}
\int_{\Omega}\left\|\varepsilon_{n}(\omega)\right\| d \mu(\omega)= & \int_{\Omega}\left\|m_{n+1}(\omega)-\left(1-\alpha_{n}\right) m_{n}(\omega)-\alpha_{n} T\left(\omega, k_{n}(\omega)\right)\right\| d \mu(\omega) \\
\leq & \int_{\Omega}\left\|m_{n+1}(\omega)-x^{*}(\omega)\right\| d \mu(\omega) \\
& +\left(1-\alpha_{n}\right) \int_{\Omega}\left\|x^{*}(\omega)-m_{n}(\omega)\right\| d \mu(\omega) \\
& +\alpha_{n} \int_{\Omega}\left\|x^{*}(\omega)-T\left(\omega, k_{n}(\omega)\right)\right\| d \mu(\omega) .
\end{aligned}
$$

Using (2.6), we have:

$$
\begin{aligned}
\int_{\Omega}\left\|x^{*}(\omega)-T\left(\omega, k_{n}(\omega)\right)\right\| d \mu(\omega)= & \int_{\Omega}\left\|T\left(\omega, x^{*}(\omega)\right)-T\left(\omega, k_{n}(\omega)\right)\right\| d \mu(\omega) \\
\leq & \left.\int_{\Omega} \| x^{*}(\omega)-k_{n}(\omega)\right) \| d \mu(\omega) \\
& -\phi\left(\int_{\Omega}\left\|x^{*}(\omega)-k_{n}(\omega)\right\| d \mu(\omega)\right) \\
\leq & \int_{\Omega}\left\|x^{*}(\omega)-k_{n}(\omega)\right\| d \mu(\omega) \\
\leq & \left(1-\beta_{n}\right) \int_{\Omega}\left\|x^{*}(\omega)-m_{n}(\omega)\right\| d \mu(\omega) \\
& +\beta_{n} \int_{\Omega}\left\|T\left(\omega, x^{*}(\omega)\right)-T\left(\omega, z_{n}(\omega)\right)\right\| d \mu(\omega) \\
\leq & \left(1-\beta_{n}\right) \int_{\Omega}\left\|x^{*}(\omega)-m_{n}(\omega)\right\| d \mu(\omega) \\
& +\beta_{n}\left[\int_{\Omega}\left\|x^{*}(\omega)-z_{n}(\omega)\right\| d \mu(\omega)\right. \\
& \left.-\phi\left(\int_{\Omega}\left\|x^{*}(\omega)-z_{n}(\omega)\right\| d \mu(\omega)\right)\right] \\
\leq & \left(1-\beta_{n}\right) \int_{\Omega}\left\|x^{*}(\omega)-m_{n}(\omega)\right\| d \mu(\omega) \\
& +\beta_{n}\left[\left(1-\gamma_{n}\right) \int_{\Omega}\left\|x^{*}(\omega)-m_{n}(\omega)\right\| d \mu(\omega)\right. \\
& \left.\left.+\gamma_{\Omega} \| T, x^{*}(\omega)\right)-T\left(\omega, m_{n}(\omega)\right) \| d \mu(\omega)\right] \\
&
\end{aligned}
$$




$$
\begin{aligned}
\leq & \left(1-\beta_{n}\right) \int_{\Omega}\left\|x^{*}(\omega)-m_{n}(\omega)\right\| d \mu(\omega) \\
& +\beta_{n}\left[\left(1-\gamma_{n}\right) \int_{\Omega}\left\|x^{*}(\omega)-m_{n}(\omega)\right\| d \mu(\omega)\right. \\
& +\gamma_{n}\left\{\int_{\Omega}\left\|x^{*}(\omega)-m_{n}(\omega)\right\| d \mu(\omega)\right. \\
& \left.\left.-\phi\left(\int_{\Omega}\left\|x^{*}(\omega)-m_{n}(\omega)\right\| d \mu(\omega)\right)\right\}\right] \\
= & \int_{\Omega}\left\|x^{*}(\omega)-m_{n}(\omega)\right\| d \mu(\omega) \\
& -\beta_{n} \gamma_{n} \phi\left(\int_{\Omega}\left\|x^{*}(\omega)-m_{n}(\omega)\right\| d \mu(\omega) .\right.
\end{aligned}
$$

Using (3.15) in (3.14), we have

$$
\begin{aligned}
\int_{\Omega}\left\|\varepsilon_{n}(\omega)\right\| d \mu(\omega) \leq & \int_{\Omega}\left\|m_{n+1}(\omega)-x^{*}(\omega)\right\| d \mu(\omega) \\
& +\left(1-\alpha_{n}\right) \int_{\Omega}\left\|x^{*}(\omega)-m_{n}(\omega)\right\| d \mu(\omega) \\
& +\alpha_{n}\left[\int_{\Omega}\left\|x^{*}(\omega)-m_{n}(\omega)\right\| d \mu(\omega)\right. \\
& \left.-\beta_{n} \gamma_{n} \phi\left(\int_{\Omega}\left\|x^{*}(\omega)-m_{n}(\omega)\right\| d \mu(\omega)\right)\right] \\
\leq & \int_{\Omega}\left\|m_{n+1}(\omega)-x^{*}(\omega)\right\| d \mu(\omega) \\
& +\int_{\Omega}\left\|x^{*}(\omega)-m_{n}(\omega)\right\| d \mu(\omega) \\
& -\alpha_{n} \beta_{n} \gamma_{n} \phi\left(\int_{\Omega}\left\|x^{*}(\omega)-m_{n}(\omega)\right\| d \mu(\omega)\right) .
\end{aligned}
$$

Hence, we have

$$
\lim _{n \rightarrow \infty} \int_{\Omega}\left\|\varepsilon_{n}(\omega)\right\| d \mu(\omega)=0 .
$$

This implies that the Noor-type random iterative scheme $\left\{x_{n}(\omega)\right\}_{n=0}^{\infty}$ is $T$-stable almost surely. The proof of Theorem 3.4 is completed.

Remark 3.5. Theorem 3.4 is a stochastic generalization of the deterministic results of Berinde [7, 8] and Rhoades [30, 31]. Moreover, it is an improvement on the results of Zhang et al. [37]. 
Next, we obtain the following corollary which is the results of Zhang et al. [37] as a consequence of Theorem 3.4.

Corollary 3.6. Let $(E,\|\|$.$) be a separable Banach space and T: \Omega \times E \rightarrow$ $E$ be a random operator of the $\phi$-weakly contractive type with $F(T) \neq \emptyset$. Let $x^{*}(\omega)$ be a random fixed point of $T$. Let $\left\{x_{n}(\omega)\right\}_{n=0}^{\infty}$ be an Ishikawa-type random iterative sequence as defined in (2.8) converging strongly to $x^{*}(\omega)$ almost surely, where $\left\{\alpha_{n}\right\}$ and $\left\{\beta_{n}\right\}$ are real sequences in $(0,1)$ such that $0<\alpha \leq \alpha_{n}$ and $0<\beta \leq \beta_{n}$. Then, $\left\{x_{n}\right\}_{n=0}^{\infty}$ is T-stable almost surely.

Corollary 3.7. Let $(E,\|\cdot\|)$ be a separable Banach space and $T: \Omega \times E \rightarrow$ $E$ be a random operator of the $\phi$-weakly contractive type with $F(T) \neq \emptyset$. Let $x^{*}(\omega)$ be a random fixed point of $T$. Let $\left\{x_{n}(\omega)\right\}_{n=0}^{\infty}$ be a Mann-type random iterative sequence as defined in (2.9) converging strongly to $x^{*}(\omega)$ almost surely, where $\left\{\alpha_{n}\right\}$ is a real sequence in $(0,1)$ such that $0<\alpha \leq \alpha_{n}$. Then, $\left\{x_{n}\right\}_{n=0}^{\infty}$ is T-stable almost surely.

\section{Acknowledgements}

Both authors wish to thank the Covenant University Centre for Research, Innovation and Discovery (CUCRID) for supporting their research.

\section{References}

[1] H. Akewe, G. A. Okeke, Stability results for multistep iteration satisfying a general contractive condition of integral type in a normed linear space, Journal of the Nigerian Association of Mathematical Physics, Volume 20 (2012), 5-12.

[2] H. Akewe, G.A. Okeke, A.F. Olayiwola, Strong convergence and stability of Kirkmultistep-type iterative schemes for contractive-type operators, Fixed Point Theory and Appications, 2014, 2014:45.

[3] H. Akewe, G.A. Okeke, Convergence and stability theorems for the Picard-Mann hybrid iterative scheme for a general class of contractive-like operators, Fixed Point Theory and Applications (2015) 2015:66.

[4] A. Arunchai, S. Plubtieng, Random fixed point of Krasnoselskii type for the sum of two operators, Fixed Point Theory and Applications 2013, 2013:142.

[5] Y. I. Alber, S. Guerre-Delabriere, Principle of weakly contractive maps in Hilbert spaces, New results in Operator Theory and its Applications (eds., Gohberg, I. and Lyubich, Y.), Birkhauser Verlag Basel, Switzerland, 1997, 7-22.

[6] I. Beg, M. Abbas, Equivalence and stability of random fixed point iterative procedures, Journal of Applied Mathematics and Stochastic Analysis, 2006, Article ID 23297, (2006), DOI 10.1155/JAMSA/2006/23297, 1-19. 
[7] V. Berinde, On the stability of some fixed point procedures, Buletinul Stiintific al Universitatii Baia Mare, Seria B, Fascicola Matematica-Informatica, 18(1), (2002), 7-14.

[8] V. Berinde, On the convergence of the Ishikawa iteration in the class of quasi-contractive operators, Acta Mathematica Universitatis Comenianae, 73(1) (2004), 119-126.

[9] A. T. Bharucha-Reid, Fixed point theorems in probabilistic analysis, Bull. Amer. Math. Soc. 82(1976), 641-657.

[10] S. S. Chang, Y. J. Cho, J. K. Kim, H. Y. Zhou, Random Ishikawa iterative sequence with applications, Stochastic Analysis and Applications, 23(2005), 69-77.

[11] D. E. Edmunds, Remarks on nonlinear functional equations, Math. Ann. 174(1967), 233239.

[12] H. Engl, Random fixed point theorems for multivalued mappings, Pacific J. Math. 76(1976), 351-360.

[13] R. Glowinski and P. Le-Tallec, Augmented Lagrangian and operator-splitting methods in nonlinear mechanics, SIAM, Philadelphia, 1989.

[14] O. Hans, Random operator equations, Proceedings of the Fourth Berkeley Symposium on Mathematical Statistics and Probability, Vol. II, Part I, University of California Press, California, 1961, 185-202.

[15] S. Haubruge, V. H. Nguyen and J. J. Strodiot, Convergence analysis and applications of the Glowinski-Le-Tallec splitting method for finding a zero of the sum of two maximal monotone operators, J. Optim. Theory Appl. 97 (1998), 645-673.

[16] S. Ishikawa, Fixed points by a new iteration method, Proc. Amer. Math. Soc. 44(1974), 147-150.

[17] S. Itoh, Random fixed point theorems with an application to random differential equations in Banach spaces, Journal of Mathematical Analysis and Applications, 67(2), (1979), 261-273.

[18] M. C. Joshi, R. K. Bose, Some topics in nonlinear functional analysis, Wiley Eastern Limited, New Delhi (1985).

[19] W. R. Mann, Mean Value methods in iteration, Proc. Amer. Math. Soc. 4(1953), 506-510.

[20] C. Moore, C. P. Nnanwa, B. C. Ugwu, Approximation of common random fixed points of finite families of $N$-uniformly $L_{i}$-Lipschitzian asymptotically hemicontractive random maps in Banach spaces, Banach Journal of Mathematical Analysis 3 (2009), no. 2, 77-85.

[21] M. A. Noor, New approximation schemes for general variational inequalities, J. Math. Anal. Appl. 251(2000), 217-229.

[22] G. A. Okeke, M. Abbas, Convergence and almost sure T-stability for a random iterative sequence generated by a generalized random operator, Journal of Inequalities and Applications (2015) 2015:146.

[23] G. A. Okeke, J. K. Kim, Convergence and summable almost T-stability of the random Picard-Mann hybrid iterative process, Journal of Inequalities and Applications (2015) 2015:290.

[24] J. O. Olaleru, A. A. Mogbademu, On the stability of some fixed point iteration procedures with errors, Boletin de la Asociacion Matematica Venezolana, Vol. XVI, No. 1(2009), 31-38. 
[25] M. O. Olatinwo, Some stability results for two hybrid fixed point iterative algorithms of Kirk-Ishikawa and Kirk-Mann type, Journal of Advanced Mathematical Studies, 1(1), (2008), 5-14.

[26] D. O'Regan, Fixed point theory for the sum of two operators, Appl. Math. Lett. 9(1996), $1-8$.

[27] N. S. Papageorgiou, Random fixed point theorems for measurable multifunctions in Banach spaces, Proc. Amer. Math. Soc. Vol. 97, no. 3 1986, 507-514.

[28] S. Reich, Approximate selections, best approximations, fixed points and invariant sets, J. Math. Anal. Appl. 62 (1978), 104-112.

[29] B. E. Rhoades, Fixed point iteration using infinite matrices, Transactions of the American Mathematical Society, 196 (1974), 161-176.

[30] B. E. Rhoades, Fixed point theorems and stability results for fixed point iteration procedures, Indian Journal of Pure and Applied Mathematics, 21(1), (1990), 1-9.

[31] B. E. Rhoades, Fixed point theorems and stability results for fixed point iteration procedures II, Indian Journal of Pure and Applied Mathematics, 24(11), (1993), 691-703.

[32] B. E. Rhoades, Some theorems on weakly contractive maps, Nonlinear Analysis, 47(2001), 2683-2693.

[33] N. Shahzad, S. Latif, Random fixed points for several classes of 1-Ball-contractive and 1-set-contractive random maps, Journal of Mathematical Analysis and Applications 237(1999), 83-92.

[34] A. Spacek, Zufallige gleichungen, Czechoslovak Mathematical Journal, 5 (1955), 462-466.

[35] H. K. Xu, Some random fixed point theorems for condensing and nonexpansive operators, Proc. Amer. Math. Soc. 110(2),(1990), 395-400.

[36] S. S. Zhang, Fixed point theory and applications (in Chinese), Chongqing Publishing Press, Chongqing (1984).

[37] S. S. Zhang, X. R. Wang, M. Liu, Almost sure T-stability and convergence for random iterative algorithms, Appl. Math. Mech. Engl. Ed., 32(6), 805-810 (2011). 